Víctor Manuel Avilés Hernández*

\title{
Relación entre el sistema electoral y el sistema de gobierno en el contexto del actual debate social y constitucional
}

* Profesor Asociado de Derecho Constitucional, Universidad de Chile.
$\mathrm{E}_{\mathrm{n}}$ la actualidad existe un debate institucional inmediato, relativo a las modificaciones al sistema electoral, y uno mediato, propio de un eventual reemplazo de la Carta Fundamental. Lo anterior, se da en un entorno de altas expectativas políticas, desapego a las instituciones y debilidad de las asociaciones. En esta situación, creemos que no considerar los efectos de estos cambios en el sistema de gobierno -sobre lo que no hay mayor debate-, podría poner en riesgo la gobernabilidad y, finalmente, la democracia representativa.

\section{Introducción}

Por estos días se insiste en la necesidad de modificar el sistema electoral. Somos de la opinión que dichas reformas deben estudiarse considerando, a lo menos: i) los efectos que las mismas podrían tener en otros elementos del diseño institucional como, por ejemplo, el sistema de gobierno, y; ii) su entorno de aplicación concreta en lo social, político y económico. En este análisis conjunto se centra esta presentación.

Como es sabido, el sistema de gobierno presidencialista chileno sigue la tendencia latinoamericana de reforzar el sistema de gobierno presidencial de los Estados Unidos de América. Lo anterior, sobre la base que el sistema americano estabilizaría las reglas y fomentaría un debate centrípeto y no polar. Atendido que a diferencia del país del norte, donde el sistema electoral parlamentario es mayoritario, el sistema chileno tiende a la proporcionalidad, se debe compensar lo anterior potenciando el rol del Presidente de la República con un incremento de las facultades legislativas, lo que, supuestamente, facilitaría la implementación de su programa de gobierno (gobernabilidad). Así, el sistema de gobierno nacional (presidencial reforzado) ha funcionado razonablemente bien como contrapeso de un sistema electoral parlamentario que se comporta, de alguna forma, con cierta proporcionalidad. 
En nuestro entorno, el debate público hoy no se refiere mayormente a cambios profundos al sistema de gobierno (más allá de las aulas) sino sólo en dos niveles de discusión, separados en el tiempo, a saber: i) el inmediato, referido al sistema electoral, aisladamente, y; ii) el "mediato", relativo a la abrogación y reemplazo de la Constitución y el mecanismo a usar al efecto (por ejemplo, la asamblea constituyente). En cuanto a contenidos se busca, principalmente, repensar rol del Estado en la sociedad y la economía; rebajar o eliminar las supra mayorías o reducir las materias a las que se aplican; reconocer el carácter multicultural y/o multinacional de Chile; incrementar los mecanismos de participación directa ciudadana, como referendos o iniciativa popular de leyes; atenuar el presidencialismo, y; modificar la regulación de ciertos derechos y libertades de corte individual; garantizar derechos civiles, culturales, sociales y económicos, etc.

Por ello, asumiendo que la tendencia en lo inmediato es, en general, avanzar hacia un sistema electoral más participativo e inclusivo (por ejemplo, de tipo proporcional en materia electoral parlamentaria) y existiendo semejantes expectativas de cambios constitucionales consecuenciales, basado en un diagnóstico actual de Chile, propongo realizar un análisis del sistema de gobierno necesario para acompañar el cambio de sistema electoral.

En la primera parte de esta exposición haré un diagnóstico de la situación actual, donde me centraré en los aspectos negativos (los hay, ciertamente, positivos) y relevantes al análisis. En una segunda etapa, analizaré posibles escenarios ante una combinación de eventuales cambios jurídicos, políticos y económicos. Finalmente, propondré un curso de acción.

\section{Descripción de la situación actual}

En lo social, en la actualidad se detecta:

I) Una gran disociación o pérdida del sentido de nación o comunidad, con derechos y deberes y como base de una república. Ello implica, por ejemplo, que hoy no sólo se habla de voto voluntario sino de incentivos económicos a votar. Ante ello, es legítimo preguntarse ¿dónde queda la idea de mejorar la calidad de la política que se usó para llegar al sistema actual?

II) La existencia de ánimo asociativo sólo en base a la defensa de intereses específicos. Estos intereses se encuentran dirigidos, mayormente, a exigir derechos o, en su caso, a rechazar deberes o costos. Lo anterior, unido a la poca capacidad de organización y persistencia de las asociaciones y a la nula posibilidad de las mismas de exigir compromisos estables. Por su parte, es posible argumentar la inexistencia de acuerdos reales en estas formas de asociación sobre proyectos comunes, concretos e implementables, pues ellos siempre exigen una transacción que no se asume a nivel individual. 
III) Un bajo umbral de costos para convocar masivamente vía redes sociales. Ello implica una alta eficacia de las asociaciones inestables, antisistémicas o de corte difuso, esto es, metodológicas pero no necesariamente conformadas en torno a propuestas específicas, de fondo y coincidentes. Acá, por ejemplo, llamamos la atención sobre movimientos como los estudiantiles o el AC.

IV) Que la familia y el Estado han dejado de ser los que proveen a la satisfacción de las necesidades públicas. Para muchos, dicha satisfacción es consecuencia sólo del trabajo individual y, principalmente, de su capacidad de endeudamiento. Así, existe un alto grado de angustia individual (o menos felicidad), alienamiento temporal (fundado en la necesidad única de conservar un empleo) y potencial tensión social en caso de incremento del desempleo. Surgen posibles clientes cautivos de políticas populistas y grupos de la población que, no obstante estar pasivos hoy, tienen un gran potencial desestabilizador ante una crisis.

En lo político creemos que existe:

I) Un alto -en realidad, desmedido- empoderamiento de minorías ejerciendo la fuerza "en la calle". Así, Incluso personas electas dentro de las instituciones democráticas (v. gr., el Congreso) anuncian que ejercerán "presiones”. Este es el caso, por ejemplo, de ciertos diputados asociados a movimientos estudiantiles.

II) Un grave desconocimiento sobre la institucionalidad vigente producto, en parte, de la desaparición de la educación cívica; de las tensiones de los años setenta traducidas en temor al diálogo como fuente de polarización; de la percepción de ineficacia del trabajo político, y; del endiosamiento del mercado como único mecanismo de satisfacción de todas las necesidad humanas. La crítica a la institucionalidad opera, en buena parte, en este ambiente de desconocimiento. Lo anterior es aprovechado por académicos y grupos específicos para generar altas expectativas en un cambio constitucional precipitado.

III) Pese a su importancia para la mejor operación del diseño institucional, existe una pérdida de representatividad de los partidos políticos. La importancia de los partidos políticos como elementos de la democracia es consecuencia de ser instancias para formar ciudadanos y, principalmente, generar grupos de pensamiento y negociación ordenados bajo las reglas legales del patrocinio de campañas (en enero de 2014, estas reglas han sido violentadas por diputados militantes que renuncian a sus partidos aún antes de ser proclamados). Esta pérdida de relevancia a sido consecuencia de que los partidos políticos son: a) percibidos como obsoletos, ineficientes y carentes de contenidos técnicos y/o capacidad de respuesta, y; b) considerados como elitistas y lejanos, cuando no, como medios de protección de intereses específicos. En este escenario, por ejemplo, puede caerse en la tentación de un gobierno de "técnicos" (con presidencia de los partidos) o, en el opuesto, de representantes de grupos específicos de presión sin una visión 
sistémica o global, con riesgo de presionar por una mala e injusta repartición de los recursos escasos.

Finalmente, en lo jurídico-constitucional y planteándose desde ya la posibilidad que exista un rezago evolutivo, vemos que:

I) Se mantiene un sistema de democracia representativa con base en los partidos políticos. Lo anterior, pese a que se clama por democracia directa y se critican a los partidos.

II) Existe un mecanismo de inscripción automática y voto voluntario. En él, la alta abstención puede ser efecto de la inercia de un sistema en el que, institucionalmente, parecía que no importaba quien gobernare. Lo anterior, pese a que la elección del 2013 podría definirse como la primera en veinte años (desde 1993) en donde, con claridad, existen modelos antagónicos en juego y se deja de lado el sistema de consensos o, si se quiere, de "toma de banderas".

III) Existe un sistema electoral parlamentario que tiende a una competencia centrípeta (incentivo marginal a la creación de grandes bloques y a la moderación del discurso) pero "bitonal", en cuanto no se asegura el "gobierno de los mejores sino de los mismos". Lo anterior puede estimarse que ha resultado positivo, salvo por: a) el descrédito de los partidos; b) la persistencia de los “mismos" partidos (salvo excepciones más bien recientes), y; c) la poca respuesta para hacer frente al fenómeno de la asociatividad de intereses específicos y para incorporar grupos de presión.

IV) Rige un sistema de gobierno presidencialista (presidencial potenciado con las amplias funciones legislativas del Presidente de la República), fuertemente asentado en un sistema de elección presidencial coincidente con el de elección parlamentaria (lo que tiende a incrementar el poder del ejecutivo) y con alto roce del ejecutivo y el legislativo en el ejercicio de la función legislativa. Además: a) se percibe la ausencia de "fusibles" institucionales, lo que en caso de crisis podría llevarla al extremo; b) el camino de crecimiento de la oposición es de "suma cero", es decir, simplemente desacreditar al ejecutivo (afectar figura del Presidente de la República) y paralizar su agenda legislativa; c) luego de la última elección y atendido sus resultados, puede darse una "exclusión de la minoría” y operarse en prescindiendo de consensos en la actividad ejecutiva-legislativa. Lo anterior podría traducirse en una mayor polarización.

Como hechos relevantes llamamos la atención sobre:

I) La baja participación en los procesos electorales, lo que -por lo pronto- nos lleva a plantearnos si son menos representativas las autoridades y si hay señal de que se quieren menos o más cambios, por ejemplo;

II) La radicalización del discurso político, algo mitigado por el sistema de la segunda vuelta presidencial; 
III) La aparición (y desaparición) de colectivos o agrupaciones críticas del sistema pero carentes de interés en asumir compromisos permanentes.

Ante de entrar en el campo de las predicciones, hacemos presente que surgió un catalizador inesperado (no el única causante) de la situación que se ha descrito: El voto voluntario.

Como elementos negativos de este sistema, estimamos que:

I) El mismo ofrece un argumento adicional para la "sociedad de derechos sin obligaciones", es decir, sin vínculo mutuo.

II) Constituye un incentivo para la polarización y/o los ofertones a grupos de interés específico, únicas fuerzas que pueden hacer vencer la inercia y llevar a votar. Ello a consecuencia de que si el voto de menos tiene mayor incidencia, hay más propensión a participar y, en tal escenario, es más barato hacerlo en base a aspectos de interés específico que capturan adhesiones en base a una posible retribución no difusa.

III) No garantiza la coincidencia política entre la mayoría parlamentaria y el Presidente (segunda vuelta). Lo anterior puede, además: a) Crear un eventual problema de gobernabilidad y, por ello, de frustración colectiva y cinismo. Por ejemplo, Argentina tiene sistema presidencial de elección mayoritaria y sistema proporcional de elección parlamentaria; b) Como respuesta a lo anterior, puede ser que (1) se apele al ofrecimiento de beneficios, legales o no, a determinados parlamentarios como base de la formación de las mayorías necesarios; o (2) surja un caudillo que clame por un sistema de democracia directa o plebiscitaria para sobrepasar la institucionalidad y sus mecanismos corruptos o ineficientes. Ya en Chile se han planteado públicamente verdaderas violaciones al estado de derecho como, por ejemplo, el mecanismo de dictar un decreto llamando a un plebiscito inconstitucional y la abstención del cumplimiento del deber de impugnación que pesa sobre los parlamentarios.

IV) Fomenta la atomización parlamentaria y el desmedido y creciente poder de parlamentarios díscolos o independientes (disociados).

\section{Escenarios que se presentan como posibles ante este diagnóstico}

Sea por cambios culturales, sea por cambios normativos, lo que ayer permitía alcanzar ciertos objetivos (sistema de gobierno-gobernabilidad y sistema electoral-moderación del debate) hoy puede ser un obstáculo a la obtención de los mismos y el catalizador de una crisis.

En una primera etapa, de implementarse un cambio constitucional a gran escala (sea o no con abrogación de la Carta Fundamental) y centrarse el debate en lo anterior, probablemente se acallará el ánimo académico de un sector. No obstante, si ello no 
se traduce en cambios concretos a la calidad de vida de la población -para mejor, se entiende-, pueden darse dos escenarios:

I) Si no hay crisis económica, parte de los grupos de la calle se sumará a la población mayormente alienada por el trabajo para sustentar el nivel de endeudamiento (y de vida), de forma similar a lo ocurrido luego de la década del ochenta, pero;

II) Si hay crisis económica y, principalmente, aumento del desempleo, se sumará la clase media intra-sistema a la calle, con grave peligro para la estabilidad institucional. En este escenario, además, se abren dos alternativas: a) si el Congreso tiene una mayoría que respalde al ejecutivo en la creación de los mecanismos legales para enfrentar la crisis, es posible su desarticulación, pero; b) Si el Congreso, atomizado por el sistema electoral, no entrega el apoyo necesario al ejecutivo (sea legislando sea delegando la facultad legislativa), ante la inexistencia de un mecanismo constitucional de superación de esta crisis, puede surgir una verdadera dictadura sin sustento constitucional claro fundada en el fin de la separación de los poderes.

\section{Eventual camino a la solución}

Desde ya, creemos necesario:

I) Profundizar la enseñanza de la educación cívica, para generar una masa crítica comprometida con las instituciones o con los cambios que se les introduzcan. La asamblea constituyente sin educación cívica es un espacio elitista, donde se terminan imponiendo ideas. Por su parte, la asamblea constituyente no es el único mecanismo de asegurar cohesión y estabilidad tras un proyecto de Constitución, especialmente si no se entienden o conocen sus vericuetos, y;

II) Modificar el sistema electoral (hacia un proporcional) de manera de generar un incentivo marginal (no el único) a que "la calle" opere dentro del sistema de democracia representativa que nos rige. Lo anterior, permitirá encausar el debate constitucional y dará cuenta de una institucionalidad que refleja el cambio cultural y, partir de una nueva conformación del Congreso, contribuye a su sustentabilidad. La alternativa de sistema electoral inversa, es decir, mantener el sistema presidencial -atenuando o no el presidencialismo- con un sistema de elecciones parlamentaria mayoritaria, podría ser eficiente pero no necesariamente haría eco en el parlamento de la diversidad actual, por lo que no es garante de un sistema estable al mediano plazo.

Pero no basta con lo anterior. En los sistemas, cualquier modificación tiende a producir efectos directos o indirectos. Por ejemplo un sistema electoral más proporcional (haciendo eco del legítimo deseo de una mayor igualdad y buscando más participación) podría generar una atomización parlamentaria o, en el mejor de los casos, mayorías parlamentarias menos estables, e, incluso, un incentivo a la corrupción. Existe doctrina 
sobre estos incentivos a la corrupción que operan en la combinación de un sistema de elección presidencial mayoritario y de elección del parlamentaria proporcional. ${ }^{1}$ Además, podría producirse una crisis constitucional por la inexistencia de respaldo de la mayoría parlamentaria al Presidente, como fue el caso del Presidente Allende y su minoría en Congreso. ${ }^{2}$

Así, es legítimo preguntarse si cambiando el sistema electoral por uno más proporcional funcionará bien el actual sistema de gobierno o deben pensarse ajustes. En un escenario de mayor representatividad en el Congreso ¿Podrá el Presidente obtener la aprobación de las leyes que aseguren plasmar su proyecto? De caso contrario ¿terminará de caer la confianza ciudadana en la democracia representativa? En esta nueva realidad, ¿a qué costo podría lograrse la gobernabilidad?, ¿habría riesgo de tiranía, corrupción o, en el mejor de los casos, de clientelismo en favor de grupos específicos.

Por ello, creemos que es necesario estudiar el efecto que la modificación del sistema electoral puede tener en la relación del Congreso con el Presidente de la República y eventualmente introducirse cambios. Al respecto, conocernos dos grandes alternativas viables en Chile:

1. Establecer un sistema realmente presidencial, sustrayendo poderes al Presidente de la República en materia legislativa pero en base a un sistema electoral parlamentario mayoritario uninominal de elección conjunta del ejecutivo, lo que no parecería recoger el sentir mayoritario de la población, o;

2. Buscar un sistema que comprometa al Congreso Nacional con el éxito del Gobierno, para favorecer la gobernabilidad. En el estudio de esta segunda línea nació la idea de buscar atenuaciones al sistema presidencial, por ejemplo, similares al sistema francés que surge en 1958, luego de un sistema parlamentario y de cierto estancamiento, todo ello en base a: a) la creación de un cargo de Jefe de Estado electo, con atribuciones en materia de relaciones internacionales; de mediación entre el ejecutivo y el Congreso y, además, de ombudsman; sin iniciativa parlamentaria pero con la facultad de enviar mensajes a través de un parlamentario. Esto reforzaría el sentido de autoridad y existiría un árbitro ante crisis institucionales; b) La creación de un cargo del Jefe de Gobierno, representativo de la mayoría parlamentaria, pero nombrado por el Presidente. El mismo ordenaría el debate parlamentario, canalizaría la mayoría parlamentaria en un sistema colaborativo

1 "De acuerdo con los estudios estadísticos, la peor combinación, la que más alienta la corrupción, es la de un sistema político basado en un presidente con amplios poderes y legisladores elegidos por un sistema de representación proporcional", dice Rose-Ackerman de Yale (Corrupción y gobierno: causas, consecuencias y reforma, de 1999).

2 Bruce Ackerman, de Yale y Harvard, ha señalado que "la forma más tóxica de división de poderes es la combinación constitucional de un presidente popularmente electo junto con un Congreso electo por un sistema de representación proporcional (...). Si un sistema electoral de representación proporcional es deseable, el sistema estadounidense de división de poderes no lo es, ya que el costo potencial de la representación proporcional en términos de estabilidad política del régimen es mucho más alto en los sistemas presidenciales que en los parlamentarios" (ACKerman, Bruce, La Nueva División de Poderes, Editorial Fondo de Cultura Económica, 2007, p. 40) 
y sería un fusible ante crisis institucionales. De esta forma (1) sólo el Jefe de Gobierno sería responsable ante el Congreso (moción de confianza/ censura), y; (2) en caso de crisis -por ejemplo, imposibilidad de formar gobierno estable- el Presidente de la República podría disolver el Congreso. De esta forma, la misma institucionalidad podría hacerse cargo de una crisis, sin deslegitimarse y convocando a la ciudadanía.

\section{Conclusiones}

A modo de conclusión:

1. El diseño institucional debe reflejar el sentimiento mayoritario de la población;

2. El cambio en un elemento del sistema institucional (sistema electoral) tiene, necesariamente, efectos en otros aspectos (sistema de gobierno);

3. La relación de los diferentes elementos de un sistema institucional debe medirse, además, en conjunto con el sustrato social y cultural imperante;

4. En términos generales, sea en pro de la estabilidad (institucionalizar la "calle" o darle número a los grupos vociferantes) sea en pro del sentimiento colectivo, todo indica que se avanzará a un sistema electoral más proporcional.

5. Dicho sistema proporcional operará junto con un sistema de gobierno presidencial “presidencialista”, donde gran parte del esfuerzo del Presidente de la República se centrará en la aprobación de leyes para plasmar su programa.

6. Luego, es probable que la atomización parlamentaria afecte la gobernabilidad (posibilidad de sacar adelante el programa presidencial) con gran desgaste de la figura del Presidente de la República, o, creando incentivos a la corrupción o a cambios fuera del sistema, especialmente en eventos de crisis económica.

7. Así, creemos necesario estudiar en conjunto el sistema electoral proporcional (asumiéndolo como un dato) ciertas modificaciones al sistema de gobierno de manera de: i) proteger la autoridad presidencial (base de la institucionalidad); ii) permitir la colaboración entre el Congreso y el ejecutivo, y; iii) contar con un sistema de solución institucional de conflictos, con posibilidades de recurrir a la ciudadanía. 